\title{
ANTROPOLOGÍA
}

\section{ANÁlISIS DE LAS CAUSAS DEL BAJO NIVEL DE CUMPLIMENTACIÓN DE LA HOJA DE VALORACIÓN DEL PACIENTE AL INGRESO: UNA APROXIMACIÓN DESDE LA PERSPECTIVA CUALITATIVA.}

Gabaldón Bravo EM.**, Gómez Robles FJ.*, Fernández Molina MA.**, Domingo Pozo M.*, Torres Figueiras M.*, Terrés Ruiz C.*, Gomis García, L.*. *Enfermeros asistenciales Hospital General Universitario de Alicante. **Director de Enfermería HGUA

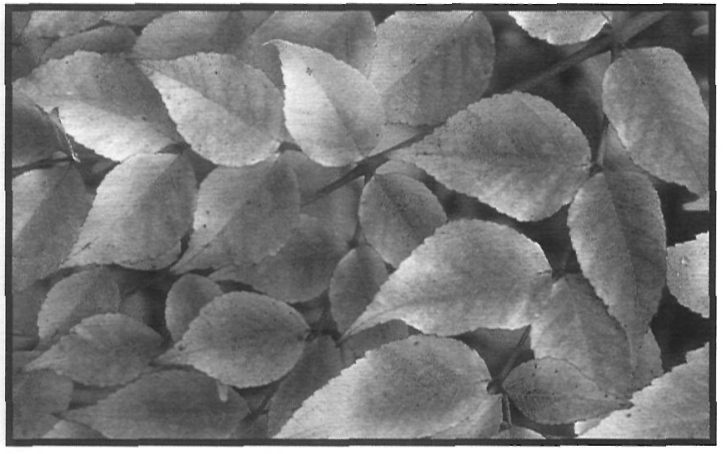

ANALYSIS OF CAUSES FOR NOT FILLING IN THE NURSING EVALUATION SHEETS ON PATIENTS'S BEING ADMITTED TO HOSPITAL: A QUALITATIVE PERSPECTIVE.

\section{SUMMARY:}

7 he current article analyses qualitatively the project: "Study of causes for not filling in 1 the nursing evaluation sheets on the patient's being admitted to hospital. Diferent perspectives to modify behaviours". (FIS 00/1049).

The project is under study by a group of hospital clinical nurses and adminstration nurses. Aims being 1: Examining index of nursing evaluating sheets presented. 2: Evaluating different measures to increase the so low index.

The methodology shows a descriptive way to quantify the above mentioned index by means of a transversal study of the clinical histories and analysis of the prior situation. A wide range of information will be requested from professionals using the technique of a nominal group. Reasons for the principal cause: sheets not being filled in should be given priorization. Strategies of intervention to solve the problem should be then taken into consideration. A pilot unit will be firstly analysed and results will be generalized to other hospital areas. Six months later, a six-month-evaluation will be done. First item to be considered is: the official sheet provided by the Consellería de Sanitat on patient's admission to hospital. Professionals from the Hospital General Universitario de Alicante - nurses - will be secondly considered. Variables to be discussed are: administrative data, general health data, basic needs: elimination patterns, mobility, feeding, respiration, hygiene... and other needs as safety, communcation, etc..

\section{INTRODUCCIÓN:}

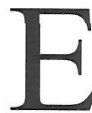

ste artículo versa sobre la parte realizada mediante técnica cualitativa del proyecto "Estudio del grado de cumplimentación de la hoja de valoración del paciente al ingreso por el personal de enfermería: evaluación de las distintas alternativas de mejora."(FIS 00/1049) y que llevan a cabo un grupo de enfermeros asistenciales y de gestión del hospital. Como indica este proyecto en su memoria, el objetivo es "comprobar el nivel de cumplimentación de la hoja de valoración del paciente al ingreso por el personal de enfermería del H.G.U.A. y sus determinantes. Evaluar diferentes medidas de intervención para mejorar el nivel de cumplimentación de la hoja de valoración del paciente al ingreso." En 
cuanto a la metodología, indica que se trata de un "estudio descriptivo para cuantificar el nivel de cumplimentación de la hoja de valoración de enfermería al ingreso mediante estudio trasversal de las historias clínicas, como análisis de la situación previa. A través de una técnica de grupo nominal, se obtendrá información de los profesionales sobre los determinantes de la cumplimentación, para la detección de las causas del bajo nivel de cumplimentación y priorización de la causa principal; posteriormente elaboración y aplicación de estrategias de intervención sobre los mismos, primero en una unidad piloto y después se generalizará a todo el hospital. Evaluación a corto plazo y trascurridos 6 meses de la intervención. El sujeto a estudio en primer lugar, es la hoja oficial de la Consellería de Sanitat para la valoración del paciente al ingreso en planta por parte de enfermería y en segundo lugar, los enfermeros del Hospital General Universitario de Alicante. Las variables a estudio son: datos administrativos, datos de salud general, necesidades básicas (eliminación, movilidad, alimentación, respiración, higiene) y otras necesidades como seguridad, comunicación, etc.

En enero del año 2000, se llevó a cabo el estudio transversal de 596 hojas de valoración obtenidas de las Historias Clínicas de otros tantos episodios de hospitalización. Para ello, el equipo de investigación diseñó previamente una planilla de recogida de datos. Tras el análisis estadístico de los resultados se confirmó la baja cumplimentación de las hojas, y se procedió con el segundo objetivo: conocer las causas de la baja cumplimentación detectado, y que es el que incumbe a este articulo

- Objetivo del estudio: Conocer las causas de la baja cumplimentación de la hoja de valoración de enfermería al ingreso en el H.G.U.A. y posibilidades de intervención que repercutan en su mejora.

\section{METODOLOGÍA:}

Diseño inicial: Mediante una técnica cualitativa de consenso (grupo nominal) se determinarán las causas que provocan el bajo nivel de cumplimentación.

Se escoge el grupo nominal debido a que identifica problemas y los clasifica en orden de impor- tancia; también combina reflexión en silencio (útil para generar ideas) e interacción (útil para evaluar).

De acuerdo con la técnica del grupo nominal, realizamos una sola sesión con una duración de 2 horas, el día 17 de diciembre, con la participación de 8 profesionales de enfermería cuya labor se desarrolla en las unidades de hospitalización escogidas De ellos, cinco eran mujeres y tres hombres, debido a que en nuestro colectivo predomina el sexo femenino. Se tuvo en cuenta a la hora de la selección características como la edad y la formación académica de los participantes. Se escogieron de diversos intervalos de edad para obtener una adecuada representación de cada uno de ellos. Así mismo se seleccionaron ATS y DUEs debido a que esta situación académica está presente en nuestro colectivo. Dentro del desarrollo de la técnica, se redactó la cuestión a debatir, la cual fue:

"¿Cuál es, según su opinión, la principal causa que provoca el bajo nivel de cumplimentación de la Hoja de Valoración del Paciente al Ingreso?"

El animador del grupo nominal fue un experto en trabajos y terapia de grupos. De aquí se esperaba, siguiendo el diseño inicial, obtener las causas y pasar al siguiente apartado del proyecto FIS, la intervención, pero dadas las conclusiones a las que se llegó, se realizó un cambio en el diseño inicial que produjo la ampliación de la consulta y el estudio de los profesionales respecto al problema detectado. Ésta vez, mediante grupos de discusión que aclararon y profundizaron en cada una de las causas detectadas y sus posibles soluciones. Cada grupo de discusión estaba formado por 8 enfermeros asistenciales, a los que se les propuso el tema a debate:

1.- Baja autoestima del profesional de enfermería.

2.- Falta de conocimientos para plasmar por escrito nuestro trabajo.

3.- Inadecuada infraestructura en las unidades y falta de tiempo.

4.- Inadecuado formato de la hoja de valoración vigente.

Al igual que en la ocasión anterior los grupos fueron llevados a cabo por profesionales de enfermería formados para ello. Todas las sesiones fueron grabadas para su posterior análisis. 


\section{Diseño final:}

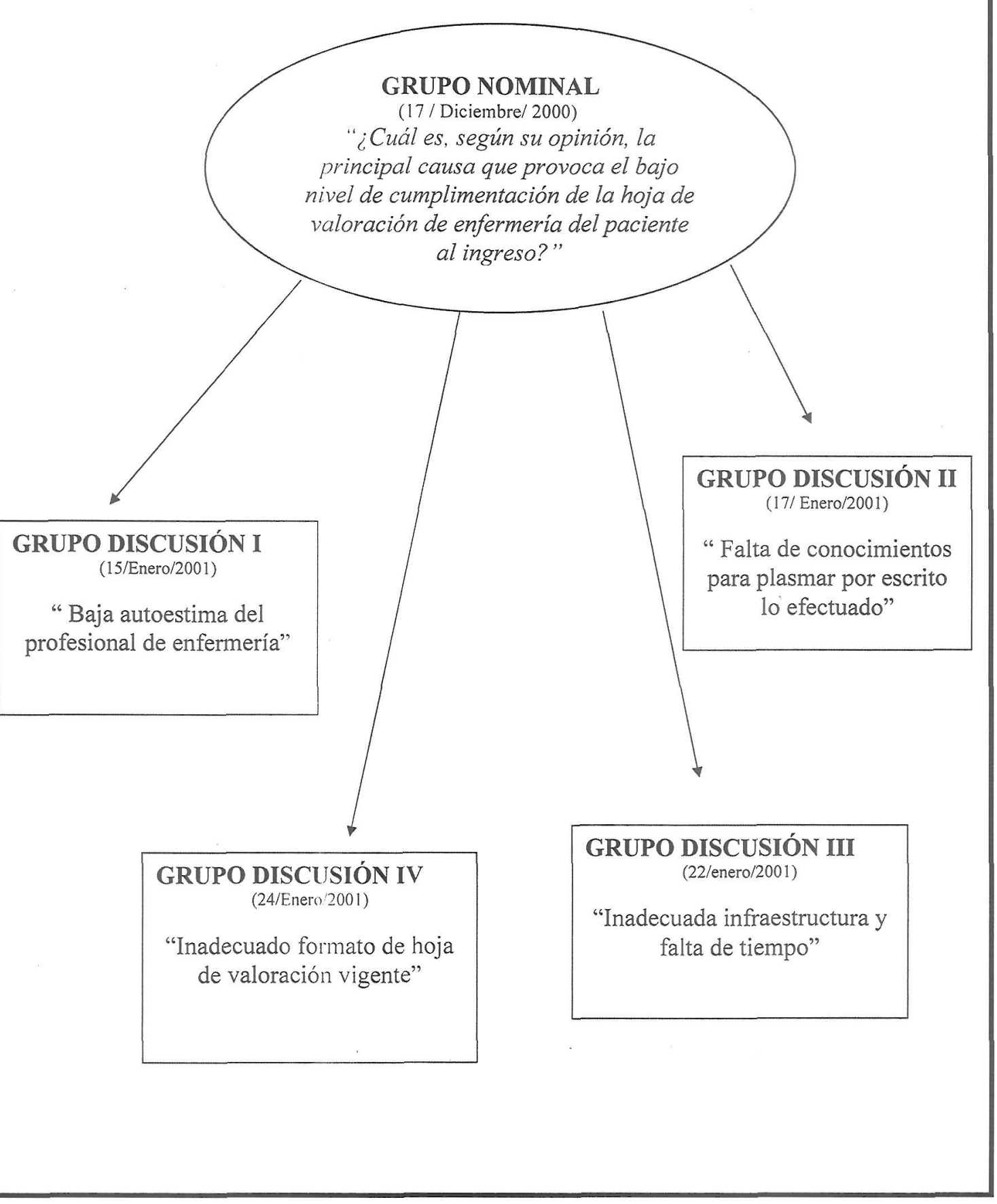




\section{RESULTADOS}

\section{1.- DEL GRUPO NOMINAL:}

-Las causas de la baja cumplimentación, por orden de importancia definidas fueron:

1) Minusvaloración de las tareas propias, tanto por parte de los agentes externos, como por parte de los propios compañeros y también uno mismo. Siempre se antepone la presión de las tareas delegadas que dificulta la realización de las tareas propias de enfermería.

2) Dificultad en plasmar por escrito nuestro trabajo por falta de costumbre y desconocimiento del lenguaje apropiado.

3) La infraestructura de las plantas no favorece las "tareas intelectuales".

4) Sobrecarga asistencial que deja a la enfermera sin tiempo para sentarse a escribir.

5) Formato de hoja no facilitador; sería preferible una hoja semiestructurada.

6) Ausencia de refuerzo / motivación (según servicios).

\section{2.- DE LOS GRUPOS DE DISCUSIÓN:}

1) Autoestima: No hay criterios unificados para la valoración, sería buena la unificación de parámetros para todo el hospital, tanto por medio del uso de una misma hoja de valoración en todas las unidades como por medio de una guía para su cumplimentación.

Se plantea la razón de separar registro de enfermería de la Historia general del paciente, por comodidad para las enfermeras; si se une a la historia general, escribirán menos (menos accesibilidad a la historia en cualquier momento)

Hay muchas enfermeras que escriben, pero lo hacen de forma individual, se han enseñado a sí mismas, y a las que no lo hacen no se les puede decir nada, puesto que no les gusta escribir, porque les cuesta... no lo viven como algo necesario, y como no se les da bien, no lo hacen. Algunas solo escriben el relevo y sólo lo más llamativo, que luego puede dar problemas, para "cubrirnos las espaldas". "No sentimos lo realmente importantes que somos... sin nosotros no habría un seguimiento individualizado y continuo del paciente."
La verdad es que lo que se escribe no se utiliza, "no vale para nada", "no lo ve nadie", "siempre te tienes que fiar de lo que haces tú, a saber lo que hace el otro turno..." es difícil convencer a la gente de que escriba cuando en realidad no sirve para nada. Sin embargo es muy importante saber todo sobre el paciente, "a ver, sino, cómo sabes cómo te llega un paciente que te trasladan de otra planta..." "Entonces, sí que es necesario escribir para que lo lea el compañero..." "Eso es facilitarle la labor al compañero" "Todos escribimos, aunque sea poco, en el relevo de enfermería, para el seguimiento del paciente por el turno siguiente, para facilitar el trabajo" (Tras un silencio de reflexión) "La enfermera siempre se ha caracterizado por proteger al paciente, y el médico por protegerse a sí mismo... debemos hacer que cambie el chip de la enfermería un poquito hacia nosotros mismos, pero sin perder de vista al paciente... la dirección debería dar soporte, crear foros de debate, hacer algo para que le enfermera se sienta segura, que su trabajo tiene una línea de seguridad y continuidad." . "Cuando uno deja por escrito lo que valora, o lo que hace con el paciente, evita los errores orales y los malos entendidos porque por escrito se tiene más precaución, por lo tanto somos más eficaces al disminuir los errores".

"Pero hay algo más, hay que creer en lo que estás haciendo, hay que darse cuenta del valor del reconocimiento personal y profesional" "Muchas veces no hay interés en empezar a escribir, porque la enfermera ha sido autodidacta en cuanto a los registros, por lo menos nuestra generación, las más jovencitas ya no tanto, auque luego las devora la rutina del trabajo en planta". "Mira, otra cosa es que, o escribimos lo que hacemos, o cómo reivindicamos las cargas de trabajo, no se refleja lo que se hace, sólo sabe lo que hemos hecho el paciente, porque no está escrito para recuperar la información" " Es la base de la investigación para nuestra profesión, y la única forma de protección legal"

"Nos falta cultura de grupo, sentarnos a mirarnos las caras, y a hablar de nuestro trabajo, llámalo sesiones de enfermería, o como quieras, pero cosas como las de hoy nos hacen falta en el hospital, pero mucho".

2) Conocimientos: "Nunca han tenido demasiado interés en que sepamos escribir bien o en que usemos una terminología culta, pienso que, para el 
estamento médico, el que no sepamos demasiado, nos hace más obedientes" "La enfermería ha sido hasta hace poco autodidacta, y eso no está bien" "perdona, pero si se escribe algo es gracias al esfuerzo de los antiguos titulados, que no han tenido ninguna formación y que se han esforzado por hacerlo" "Lo que está claro es que existen dos clases de enfermería, la de las teorías, que me parecen muy bien, que es la de la investigación, la que está en la universidad, y luego la que trabaja a pie de cama, que no entiende de filosofías ni de investigación, y que como mucho, ha oído hablar de Florence Nightingale, o de Henderson, por lo de las necesidades que todos más o menos manejamos, pero que no les pidas nada más... y eso no quiere decir que sean malos profesionales, hay gente muy buena que no sabe escribir y no lo hace, y punto".... "Tenemos que ponernos de acuerdo en un lenguaje claro, nuestro, que nos ayude a comunicarnos... mira como en los relevos orales todos nos entendemos, pues igual pero por escrito"... “ La única forma de solucionar esto es mediante una buena oferta de formación continuada y continua en registros, pero a la que asistamos los enfermeros....y para eso hay que estar motivado".

"La gente se queda tan tranquila pensando que rellena las hojas, como por trámite, y ojo que les digas algo, aunque entre otras cosas, las viejas glorias, ......, que son las autodidactas, se sienten en poder de la verdad absoluta....yo no se si es por eso o porque están quemadas, pero no hay quien las haga cambiar, al contrario, si te ven sentada escribiendo, te pegan cada mirada que..." "Pues la nueva hornada de DUEs terminará haciendo lo mismo, porque el trabajo te come, aunque es cierto que su formación no es la nuestra, tendréis que esperar a que nos jubilemos..." "Hablando en serio, una hoja semicerrada, donde se pudiera anotar de forma sencilla, facilitar el paso intermedio, aparte de la formación, ¿No sería adecuado a nuestra situación?" "Pues sí, porque a veces cuando utilizamos algún término, yo entiendo una cosa y mi compañera otra...." "O el uso de escalas, o la definición general de los términos que vayamos a emplear anotados en una guía..." "Las guías ya existen y no se las lee nadie desde el año catapún, y no hablemos de los protocolos en planta, ...(murmullos de desacuerdo)... bueno al menos en algunas plantas"
"Deberíamos actualizar todo lo que utilizamos por escrito, todos los indicadores, las escalas, aquí la que se está continuamente actualizando es la de las escaras, los supervisores se encargan a todas horas, y eso es porque nos interesa, pero solo en las plantas donde se tiene más pacientes encamados, no todo el hospital" "Que tenemos que hablar más y ponernos de acuerdo en lo que escribimos y no hay nada como hablar para entenderse".

3) Infraestructura y tiempo: "Se necesita un sitio específico donde haya tranquilidad para poder escribir, porque el control no es para escribir; suena el teléfono, te llaman de la habitación..." "Sí, pero de dónde sacas tiempo para meterte en un despacho para ponerte a escribir? Yo no puedo dejar a mi compañera sola con toda la planta durante media hora para irme a escribir" (se analiza por parte de todos la necesidad de hacer una buena valoración del paciente al ingreso en planta) "Se debe estar más tiempo con los enfermos" "Se necesita nuestra independencia con respecto a los médicos, porque nos delegan muchas tareas" "El descuido de los médicos acumula trabajo, y si no vas haciendo de secretaria, eres desobediente...incluso en algunos servicios se les rellenan las peticiones.....la secretaria es del servicio, y no del médico." (se comenta cómo poder afrontar el problema con los médicos, y cómo lo han hecho los servicios donde ya lo han superado) "Si todos nos ponemos de acuerdo en qué tenemos que hacer y qué tenemos que tolerar, y si la dirección nos apoya el tema, se supera, lo malo es que en algunos servicios estamos desamparados y terminas pidiendo el traslado o quemándote, y siguen con el mismo tipo de enfermería que hace treinta años" "Desde siempre enfermería asume las tareas que no son de nadie, y además es que nos lucimos, lo hacemos todo estupendamente..." "La de tiempo que ganaríamos si nos quitáramos de encima las tareas administrativas; ordenar las historias de los pacientes, pedir pegatinas, reclamar analíticas, pasar visita con el médico...", "La independencia profesional se consigue si somos capaces de reivindicar nuestro tiempo para nuestras tareas y viceversa, trabajamos con método, nos motivamos si somos reconocidos por nosotros mismos....intentar introducir algo por narices es un horror..." "Cada planta, 
según su ritmo de trabajo debería tener su tiempo preestablecido... si nos centramos en la hoja de valoración, hacerla en el momento del ingreso, con el paciente, en la habitación del paciente, al mismo tiempo que te presentas, es una buena forma de entrar en contacto..." "Si tienes que escribir o anotar primero, y luego pasar a limpio pierdes tiempo" "Es necesario que todo el equipo que trabaja con el paciente comparta todos los recursos de la planta, y si hay despachos para escribir o salas para reuniones, que no ponga en la puerta que es propiedad privada de los médicos".

4) Formato hoja: Se inicia la reunión comentando la necesidad de usar un lenguaje común, propio de enfermería, "porque no somos médicos, auque nos tengamos que entender con ellos, pero como equipo" "Creo que la hoja de valoración actual es demasiado abierta, te deja en el aire.... sería bueno cerrada" "Sí, pero no cerrada del todo, que debemos manejar vocabulario... no está bien que nos dediquemos a pones cruces sin más, vamos a calentarnos un poco la cabeza..." "Vale pues con algunos puntos que sean cerrados y otros abiertos, un formato mixto" "Una guía ayudaría bastante, pero una guía que se encontrara en el mismo sitio donde haces la valoración que no tuvieras que buscarla a todas horas y que no fuera un tocho, sino algo sencillo, dos, tres folios..." "Esa guía serviría para unificar criterios y también el uso del vocabulario, con definiciones operativas de cada item,..." "Debemos acostumbrarnos a utilizar índices y escalas, incluso dependiendo de cada unidad, pero que todos los conociésemos" "Para eso sería bueno realizar sesiones monográficas de registros, para actualizar conocimientos... y así nos comunicamos cosas de nuestro trabajo,.. hace tiempo estuve en una planta donde se hacían sesiones de enfermería y era una maravilla hablar de nuestro trabajo".

\section{CONCLUSIONES:}

Tras la realización de la técnica de grupo nominal, la primera conclusión que se obtuvo, fue la necesidad de continuidad en el acercamiento a los profesionales y entre los profesionales de enfermería, que iban a ser los protagonistas, los actores del posible cambio. Si reflexionamos sobre lo ocurrido durante el grupo nominal, y los comentarios realizados por los participantes a la salida de la sesión, y también durante ella, vemos que el sentimiento de ser escuchados, de sentirse con voz, y a su vez escuchar al compañero, era un refuerzo clave para la consecución del objetivo del proyecto. De esta forma, el investigador, con el hecho de su intervención ya estaba favoreciendo un cambio, útil para su finalidad.

La necesidad, como hemos indicado, de continuidad en el hecho de servir como simple catalizador al servicio de la necesidad de cambio del profesional de enfermería, al mismo tiempo que la posibilidad de ampliar el conocimiento de campo sobre la cuestión, nos animó al cambio de estrategia, programando y planificando los grupos de discusión, que dieron entrada a 32 profesionales más.

La hoja de valoración de enfermería fue la puerta de acceso a temas claves de la praxis enfermera; cada una de las causas obtenidas, es aplicable a la generalidad, al origen de nuestra perspectiva actual, o al menos explicativa de nuestra situación de evolución profesional. No digamos de nuestra evolución científica. La división entre enfermería asistencial y enfermería teórica es un hecho tal, que en ningún momento de las discusiones de trabajo se comentó la existencia de teóricas que contemplaran modelos aplicables a la praxis enfermera que solucionaran el problema de la no escritura, o que ni mucho menos sintieran la necesidad de adoptar un modelo de enfermería como posible solución a su situación laboral, en cuanto a la relación con el paciente o con el resto de su entorno. Los profesionales reconocen su falta de formación en cuanto a la cultura propia de la profesión, con todo lo que ello conlleva en cuanto a la falta de identidad asumida. Nunca se podrá adoptar medidas en aspectos que se desconocen. Por todo ello, la adopción de un determinado modelo de registro, tendrá que adaptarse al momento profesional y a las características propias de los profesionales que lo integran; un modelo de registro abierto da pie a la no escritura en nuestro país, en estos profesionales si antes no se hace incidencia en el cambio cultural, de hábitos, de conocimientos, de vivencia del trabajo individual como parte del trabajo profesional de un colectivo que se reconoce en cada uno de sus miembros. 
En cuanto a los grupos de discusión, en primer lugar indicaremos que, al igual que a la hora de la realización del grupo nominal, todos los convocados de forma aleatoria, asistieron a su sesión (excepto en un par de casos, que justificaron su ausencia por causas personales). Destacamos esto porque es un indicio más que confirma el interés mostrado por los profesionales por el hecho de ser consultados. Todos participaron activamente, no mostrando reticencias a la participación en ningún caso, aunque sí, por supuesto, diferencias de opinión más o menos exaltadas entre los compañeros.

De forma generalizada, en los cuatro grupos de discusión los participantes manifestaron la falta de estima tanto por parte del resto del equipo de la unidad como por parte de los propios profesionales de la labor efectuada por enfermería, ante todo de las labores propias. En todos los grupos, la inadecuada formación de los profesionales "antiguos titulados" también se manifiesta, en ocasiones para defender la gran cantidad de autodidactas en registros que hay en nuestra profesión. La falta de motivación queda patente en todos los casos.

En cuanto a los resultados obtenidos en el primer grupo, que llamaremos "Autoestima", deja clara la necesidad de autovaloración del profesional y propone como estímulo para ello la reflexión "en voz alta" sobre nuestro trabajo, entre los compañeros, en sesiones de enfermería o en cualquier otro evento, de forma que se acabe con la sensación de "no valer para nada. También queda clara la necesidad de dejar por escrito lo que valora enfermería, porque "sí que valoramos al paciente, sólo que no lo plasmamos por escrito".

En cuanto a los resultados del segundo grupo "Conocimientos", se indica una situación ya conocida y vivida por los profesionales de enfermería de nuestro país; la coexistencia de distintas titulaciones, de distintas filosofías en la formación de profesionales de enfermería, y la sensación de desconexión entre el mundo teórico-científico-filosófico y el mundo laboral-práctico. Como solución se propone el esfuerzo de actualización personal, por medio de seminarios, cursos, formación continua y continuada....hablar entre los profesionales en distintos foros, de nuevo con la finalidad de sentirse escuchado, motivado.

En el grupo "Infraestructura y tiempo" se llega a la discusión del rol de la enfermera en la planta . También aparece la asunción de tareas de secretariado del médico y la sumisión a sus "órdenes". Como indica J. Luís Medina:

"Las relaciones de poder entre Medicina y Enfermería han naturalizado formas socialmente construidas de opresión transformando estructuras sociales jerárquicas y de privilegio en diferencias biológicamente determinadas lo que a obligado a las enfermeras a internalizar un estatus de sumisión respecto la medicina. Estatus que es reproducido inopinadamente en los centros asistenciales donde las enfermeras ejercen su labor." ("La pedagogía del cuidado: saberes y prácticas en la formación universitaria en enfermería. Laertes. 1999. Barcelona. pp.:131)

Una de las posibles soluciones apuntadas atañen a la gestión interna de cada planta, tomando cono ejemplo a aquellas en las que se haya solucionado de forma satisfactoria el tema. Por supuesto, que el sentirse apoyado por la dirección (supervisores, dirección de enfermería) frente al estamento médico, también es básico para los profesionales consultados. Se deduce de las últimas intervenciones que el uso de todos los recursos existentes en la planta sería suficiente para subsanar el tema de la falta de espacio, si se consigue la posibilidad de su uso por parte de enfermería y se hace un uso adecuado del mismo.

Sobre el "formato de la hoja", tema que también se trata de forma general en todos los grupos, una vez tratado de forma monográfica en el último grupo de discusión, se decide la necesidad del cambio de la hoja existente, ya que no se adapta a la situación real de enfermería en nuestro país; se demanda una hoja semiestructurada, con items cerrados alternados con items abiertos, de forma que sea mucho más operativa y clarificadora, dando paso a un lenguaje común. De nuevo queda clara la no adaptación del modelo de hoja de valoración abierta al momento profesional, en el que se necesita una mayor aportación de estructuras-guía frente a la pobreza de conocimientos en cuanto a plasmar con un vocabulario propio aquello que se realiza, e incluso frente a la necesidad de reflexionar sobre aquellas labores propias de la profesión y sobre su verdadero valor dentro del equipo de salud y frente al paciente. 


\section{0 - Cultura de los Cuidados}

Otra aportación sería la elaboración de una guía escueta, operativa que "se encontrara en el mismo sitio donde haces la valoración, que no tuvieras que buscarla a todas horas, y que no fuera un tocho... ". Enriquecer el campo terminológico, por medio del uso de índices, escalas, criterios unificados, sin perder de vista que estos deben ser impulsados, consensuados, admitidos por el profesional que los va a utilizar en su trabajo.

Todas las estrategias de mejora de la cumplimentación de la hoja de valoración (algunas de ellas ya se están llevando a cabo) nacen de estas soluciones aportadas por los profesionales. Desde luego, modificaron el proyecto inicial.

\section{AGRADECIMIENTOS:}

- D. José Sánchez.

- Dña Begoña Bebía.

- Todos los enfermeros/as del Hospital General de Alicante que participaron en los grupos de discusión y nominal.

- Unidad piloto. Servicio de Neurología del Hospital General de Alicante.

- Dña Rosario Ferrer.

\section{BIBLIOGRAFÍA:}

- Gutiérrez de Terán Moreno, G. (1999) “Aspectos legales de los registros de enfermería“.Enfermería Clínica. Vol. 3, n 1, 21-24.

- Herrero García, T. Et Alt. (1998) "Control de calidad en los registros de enfermería". Enfermería Intensiva. Enero-marzo, 9(1): 10-15.

- Córcoles Jiménez, M.P. et Alt.(1997) "El dossier de enfermería en el complejo hospitalario de Albacete: composición y calidad". Revista Rol, nº1, 19-24.

- Miró Bonet, M. ; Amorós Cerdá, M.; De Juan Sánchez, S.; Fortea Cabo E.; Frau Morro J.; Moragues Mas J.; Pastor Picornell, C.(2000) "Valoración al ingreso del paciente crítico. Un indicador de calidad asistencial". Enfermería Intensiva; 11(2): 51-58

- Marc Mandell, Jd.(1995) "Si no se ha registrado es que no se ha valorado" Nursing..

- Aparicio Casals, M.R. et Alt.(1993) "Registros de enfermería en atención primaria: pieza clave para la investigación". Enfermería Científica. Abril n 133, 11-14.
- Alonso González, M. et Alt.(1996) "Valoración de la calidad de las historias clínicas". Gestión Hospitalaria. $n^{\circ} 4,44-50$.

- González Carrión, P. Et Alt.(1998) "Validación de los diagnósticos de enfermería de la NANDA: una experiencia en cuatro áreas asistenciales" Enfermería Clínica. Vol. 4, no 3, 124-27.

- Polo Tolosana et Alt.(1999) "Registros: Revisión de la documentación clínica de enfermería de un hospital general en el año 1994". Enfermería Clínica. Vol. 7, $\mathrm{n}^{\circ} 3,72-79$.

- Blanco Rodríguez, J.M. et Alt.(1997) "Control de la calidad del registro de valoración de enfermería al ingreso". Enfermería Científica. Jul-Ago, 184-85.

- Romera Rufián, P.F.(1993) "GARE. Guía de auditoria de los registros enfermeros".Index de Enfermería, verano-otoño, año II, nº 6, 36-40.

- Amezcua, M.(1995) "La calidad en la documentación clínica de enfermería: del papeleo a los registros para el cuidado" Index de Enfermería, invierno, año IV, $\mathrm{n}^{\circ} 11,15-20$.

- Besora Torradeflot, I.(1998) "Implantación de una hoja de valoración de enfermería en Atención Primaria". Enfermería Clínica. Vol. 4, n 3, 44-46.

- Corrales Nevado, D. Et Alt. (1994) "Protocolización y registro en atención primaria: a propósito de una hoja de monitorización" Atención Primaria, marzo, 15 vol $13, \mathrm{n}^{\circ} 4$.

- Tomás Vidal, A.M. et Alt. (1992) “Desarrollo de un formato de valoración por patrones funcionales de salud".Enfermería Científica, nov, $n^{\circ} 128,18-21$.

- Marguerite V.B. et Alt. (1998) "Clinical quality measurement. Comparing chart review and automated methodologies" Medical Care.EEUU.

- Topp, M. Et Alt.(1997) "Validation of a celebral Palsy Register". Clinic Epidemiology, Vol 50 nº 9, 1017-23.

- Siles, J.(1997) “Epistemología y enfermería: por una fundamentación científica y profesional de la disciplina”. Enfermería clínica. Vol. 7, núm.4.

- Ivonna S. Lincoln, (1997) "Conexiones afines entre los métodos cualitativos y la investigación en salud": Investigación y adecuación en enfermería. Medellín, XV (2) Septiembre, pp. 57-69.

- José I. Ruiz Olabuenaga (1999) "Metodología de una investigación cualitativa. Universidad de Deusto. Bilbao.

- Pike, Kenneth.(1954) "Language in Relation to a Unified Theory of the Structue of Human Behavior". Institute of linguistics. Santa Ana. California. 